KLEIST-JAHRBUCH 1991 


\section{KLEIST-JAHRBUCH 1991}

Herausgegeben von

Hans Joachim Kreutzer

J. B. METZLERSCHE VERLAGSBUCHHANDLUNG STUTTGART 
Anschrift des Herausgebers:

Prof. Dr. Hans Joachim Kreutzer

Universität Regensburg, Institut für Germanistik

Postfach 397, D-8400 Regensburg 1

\section{Redaktion: Ernst Osterkamp}

Die Deutsche Bibliothek - CIP-Einheitsaufnabme

Kleist-Jahrbuch .../-Stuttgart: Metzler.

Erscheint jährlich. - Früher im Verl. E. Schmidt, Berlin. -

Aufnahme nach 1990 (1991)

ISSN 0722-8899

1990 (1991) -

Verl.-Wechsel

ISBN 978-3-476-00767-4

ISBN 978-3-476-03367-3 (eBook)

DOI $10.1007 / 978-3-476-03367-3$

Dieses Werk einschließlich aller seiner Teile ist urheberrechtlich geschützt.

Jede Verwertung außerhalb der engen Grenzen des Urheberrechtsgesetzes ist ohne Zustimmung des Verlages unzulässig und strafbar. Das gilt insbesondere für Vervielfältigungen, Übersetzungen, Mikroverfilmungen und die Einspeicherung und Verarbeitung in elektronischen Systemen.

(c) 1991 Springcr-Vcrlag $\mathrm{GmbH}$ Deutschland Ursprünglich erschienen bei J. B. Metzlersche Verlagbuchhandlung und Carl Ernst Poeschel Verlag GimbH in Stuttgart 1991 


\section{INHALT}

Vorwort $\ldots \ldots \ldots \ldots \ldots \ldots \ldots \ldots \ldots \ldots \ldots \ldots \ldots \ldots \ldots \ldots \ldots \ldots \ldots$

Kleist-Preis 1990

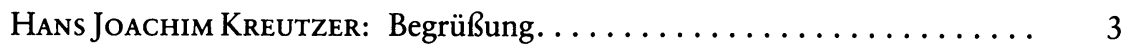

Beatrice von Matt: Heiner Müller. Rede anläßlich der Verleihung des Kleist-Preises 1990 . . . . . . . . . . . . . . . . . . .

HeINER Müller: DEUTSCHLAND ORTLOS. ANMERKUNG ZU KLEIST. Rede anläßlich der Entgegennahme des Kleist-Preises . . . . . . . .

\section{Kleist-Kolloquium Budapest 1990}

Antal Mádl (Budapest): Kleist in Ungarn . . . . . . . . . . .

HANS-JÜRgEN Schrader (Genf): Spuren Gottes in den Trümmern der Welt. Zur Bedeutung biblischer Bilder in Kleists EErdbeben.$\ldots \ldots \ldots \ldots 34$

Siegfried Streller (Berlin): Verantwortung und Verantwortlichkeit in Kleists $>$ Prinz Friedrich von Homburg.$\ldots \ldots \ldots \ldots \ldots \ldots \ldots$

Erika Tunner (Paris): Penthesilea und Penthésilée. Die Tragödie aus franzö-

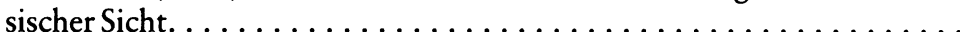

Zsuzsa SzÉLL (Budapest): Überlegungen zu Kleist - hier und heute . . . . . 67

Hans-Christian Stillmark (Potsdam): Zur Kleist-Rezeption Heiner Mül-

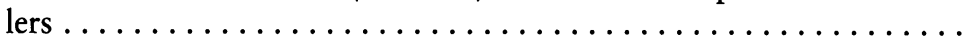

GüNTHER RüHLE (Bad Soden): Kleist - ein Autor der siebziger und achtziger

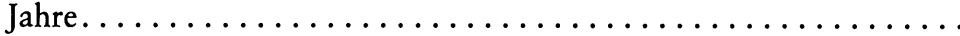

Christof Schmid (Stuttgart): Theater im deutschen Fernsehen - Kleist als Beispiel ........................... 97 


\section{Jahrestagung 1990: Mythos und Geschichte}

Uvo HölsCHER (München): Gott und Gatte. Zum Hintergrund der sAmphitryon«-Komödie.

Angelika Geyer (Bamberg): Penthesileas Schwestern. Amazonomachie als Thema antiker Kunst .

BERnd Leistner (Leipzig): Kleists politischer Furor und sein `Letztes Lied ‘ .

Bernhard Böschenstein (Genf): Der "Gott der Erde «. Kleist im Kontext klassischer Dramen: Goethe, Schiller, Hölderlin

\section{Abhandlungen}

Herbert Uerlings (Trier): Preußen in Haiti? Zur interkulturellen Begegnung in Kleists Verlobung in St. Domingo $\ldots \ldots \ldots \ldots \ldots \ldots \ldots$

SigRID WeIgel (Essen): Der Körper am Kreuzpunkt von Liebesgeschichte und Rassendiskurs in Heinrich von Kleists Erzählung >Die Verlobung in

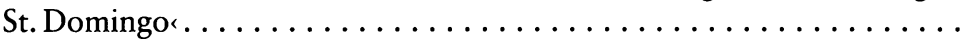

Soichiro Itoda (Tokyo): Die Funktion des Paradoxons in Heinrich von Kleists Aufsatz > Über die allmähliche Verfertigung der Gedanken beim Reden.

\section{Rezensionen}

Gerhard Schulz (Melbourne) über: Heinrich von Kleist: Sämtliche Werke und Briefe. Hg. von Ilse-Marie Barth, Klaus Müller-Salget, Stefan Ormanns und Hinrich C. Seeba. Bd. 3. . . . . . . . . . . . . .

FrIEDRICH STRACK (Heidelberg) über: Bettina Schulte: Unmittelbarkeit und Vermittlung im Werk Heinrich von Kleists . . . . . . . . . . . .

INGEBORg HARMS (Boston) über: Hans Neuenfels: Europa und der zweite Apfel. Der tollwütige Mund. 


\section{VORWORT}

Die Entstehung eines Bandes des Kleist-Jahhrbuchs nimmt durchschnittlich drei Jahre in Anspruch. Im Fall des hier vorgelegten Jahrbuchs erstreckte sich der Entstehungsprozeß über die politische Umbruchphase der Jahre 1989 bis 1991. Dieses Jahrbuch hatte damit den abenteuerlichsten Werdegang unter den zehn nunmehr existierenden.

Die zeitliche Abfolge war eine andere als die der Teile dieses Bandes. Nach Gebühr hat zuerst der Dichter das Wort, voran steht die Dokumentation über die Vergabe des Kleist-Preises im November 1990 an Heiner Müller. Man müßte den zeitgeschichtlichen Rahmen mitliefern, wenn man für den Leser, vor allem für den späterer Jahre, wenigstens andeutungsweise festhalten wollte, welche Risiken ein Redetext in einem solchen Augenblick enthielt. Die Anwesenden haben an mehr als einer Stelle der Rede Heiner Müllers den Atem angehalten: jedweder, der damals etwas öffentlich formulierte, stand wie in einer sich vorwärts bewegenden Wanderdüne.

Den ältesten Teil dieses Buches bilden die Vorträge des Budapester Kleist-Kolloquiums vom 26. und 27. April 1990. Es beschloß die vom Goethe-Institut veranstalteten Kleist-Wochen; dabei waren Filme von und nach Inszenierungen von Peter Stein, Gustav Ucicky, des Csiky-Gergely-Theaters in Kaposvá, des Nationaltheaters Weimar, von Hans Jürgen Syberberg, Claus Peymann und Hans Neuenfels gezeigt worden - weiter ließ sich der Kreis schwerlich ziehen. Nicht nur unter damaligen Bedingungen war dieses Kolloquium jedoch ein finanzieller und organisatorischer Drahtseilakt. Es steckten Probleme grundsätzlicher Art darin, die bestehen bleiben: die Realität einer literarischen Gesellschaft, was ihre Zusammensetzung und ihre Ziele angeht, kommt nicht überein mit den Vorstellungen und Arbeitsbedingungen ihrer offiziellen Geldgeber. Der Bundesminister des Innern, bisher der Hauptfinanzier für die Einzelprojekte der Kleist-Gesellschaft, hätte sich an einer Tagung an einem Ort außerhalb der Bundesrepublik, der mit Kleists Lebensweg in einer sprechenden Beziehung steht, durchaus beteiligt. In diesem Sinne ist beispielsweise von Thun in der Schweiz schon seit einem Jahrzehnt die Rede, und auf der Münchner Mitgliederversammlung 1990 wurde mit ganz besonderem Nachdruck Paris als Kleist-Stadt von hohem Symbolwert reklamiert; daß die Kleist-Gesellschaft in diesem Sinne unter den Städten der früheren DDR an erster Stelle Dresden aufsuchen würde, stand damals praktisch schon fest. Budapest hingegen, so das Bundesinnenministerium, falle in das Gebiet der auswärtigen Kulturpolitik. Diese Meinung mußten wir hinnehmen. Angeschlossen haben wir uns ihr nicht. 
Das Auswärtige Amt brauchte man nicht erst um Hilfe anzugehen, denn als Träger des Goethe-Instituts hätte es Doppelförderung nicht leisten können. - Die Deutsche Forschungsgemeinschaft schied fast ganz aus, denn ihre satzungsmäßige Klientel (Angehörige deutscher wissenschaftlicher Einrichtungen, aber nur dann, wenn sie die deutsche Staatsbürgerschaft besitzen) war nur in einem einzigen Exemplar vertreten. Immerhin, eine Fahrgemeinschaft nach Budapest ließ sich so einrichten. Es blieb als hilfreicher Partner schließlich der Deutsche Akademische Austauschdienst. Dessen Generalsekretär, Dr. Roeloffs, vermochte den Stifterverband für die Deutsche Wissenschaft zu mobilisieren, der uns Einladungen an Gäste aus der DDR ermöglichte. Ein inhaltlich gleichartiger Bittgang in Berlin, dem "Sitzland " der Gesellschaft, erbrachte nichts außer dem liebenswürdig formulierten Angebot der Senatskanzlei, Anschriften Bonner Ministerien bereitzustellen. Daß am Ende noch das Budapester Kultur- und Informationszentrum der Deutschen Demokratischen Republik drei Gäste beisteuern würde, konnten wir die längste Zeit nicht wissen; die Vorsicht dort war sehr groß gewesen.

Gearbeitet wurde in einer Dreierkonstellation von Goethe-Institut und den Universitäten in Budapest und Regensburg. Die geisteswissenschaftliche Universität, die Eötvös Loránd Universität Budapest, stellte die Räume, und Antal Mádl, der Doyen der ungarischen Germanistik, arbeitete als Konsulent mit. Ohne eine viele Jahre alte freundschaftliche Beziehung zwischen uns, entstanden bei einer Goethe-Tagung, wäre man das Wagnis vielleicht nicht eingegangen. Ursprünglich waren das Luftschlösser: zwei Universitäten in Donaustädten, Regensburg und Budapest, die Donau eigentlich ein Handelsweg des Mittelalters, es wäre doch fast natürlich, wenn man sich mit Studenten einmal besuchte usw. So kam es, daß das Germanistische Institut der Universität Regensburg - und das ist nun einmal länger als ein Jahrzehnt die praktische Basis der Kleist-Gesellschaft - die Organisation übernahm: mit ungarischen Professoren, solchen aus der Schweiz, aus Frankreich, Kalifornien, OstBerlin, Halle, Theaterintendanten aus Dresden und Frankfurt/M., einem FernsehFachmann, schließlich auch Lektoren ungarischer Universitäten und dem Direktor des Kleist-Museums in Frankfurt/O. Dies ist genau die richtige, weil lebendige Mischung, nur - für einen derart bunten Haufen ist keine Förderinstitution zuständig. Ohne das Goethe-Institut, das während der ganzen Zeit der Vorbereitung und Ausführung mit unüberbietbar vornehmer Dezenz in der Kulisse verblieb, wäre man den Realitäten der Haushaltspläne machtlos ausgeliefert gewesen.

Mit einem Thema wie Kleist war man auf dem Boden eines Ostblock-Landes in mehr als einer Beziehung im Ausland, und daran wird sich nach Lage der Dinge auf absehbare Zeit wohl wenig ändern. In der zugespitzten politischen Situation lag dann noch ein ganz besonderer Reiz des Versuchs. Es gibt viele Länder, in denen die Sicht auf den Kanon der deutschen Literatur in den letzten hundert Jahren keine nennenswerten Veränderungen erfahren hat. Das ist nicht zuletzt dem habituellen beruflichen Hochmut früherer deutscher Germanisten zu verdanken, die ihr eigenes literarisches Weltbild für gleichsam naturgegeben gehalten haben. Es war nun gerade ein Ungar, 
der über Jahrzehnte hin für die Verödung des Bildes von der deutschen Literatur eine theoretische Rechtfertigung lieferte, weit über die Grenzen des einstmaligen sowjetischen Machtbereichs hinaus. Es waren dann wieder andere, denen diese Theorie aus vielen Gründen passend erschien, so daß sie den Ideen von Georg Lukács zur politischen Durchsetzung verhalfen. Ein offener Blick auf diese Epoche: das war die Aufgabe des Budapester Kolloquiums. Die Frage nach ästhetischen und damit nach menschlichen Normen und die Einschätzung eines Falles wie desjenigen des Dichters Heinrich von Kleist als Normabweichung oder aber als eine Existenz aus eigenem Recht - das reichte noch bis tief in unsere Diskussionen vom April 1990 hinein, übrigens durchaus nicht nur unter ost-westlichen Kategorien. Es bleibt eine leise, ironische Paradoxie, daß der erste Anstoß von dem zentralen deutschen Kulturinstitut ausging, das den Namen "Goethe « trägt. Vielleicht muß man dieses Institut nicht geradezu in eine Einrichtung wider die Überschätzung Goethes umwandeln, denn auf ein verholztes, verödetes Goethebild stößt das Goethe-Institut selbst an allen Enden der Welt, aber es hat sich programmatisch längst abgekehrt von überlebten kulturellen Normsetzungen. Für seinen eigenen Neubeginn in Budapest freilich hatte der Institutsleiter, Graf Westerholt, ein ganz erhebliches Risiko auf sich genommen. Er war es, der den Einfall hatte. Ihm haben wir Dank dafür zu sagen.

In einer Atmosphäre freundschaftlicher Vertrautheit bewegte sich die KleistGesellschaft dann im November 1990 mit ihrer Jahrestagung in München, wo man über gegebene Traditionen diskutierte, über Denkformen und Bilder der Antike und über Kleist im Kontext seiner Zeit. Damit wurde bewußt ein Faden fortgesponnen, der 1985 auf dem Amazonen-Kolloquium in Berlin zuerst geknüpft worden war. Christa Wolf war im Hintergrund anwesend, sie wurde höflich befragt, aber sie wurde auch bezweifelt. Daß Uvo Hölscher sich lange schon mit der Denkweise von 'Kassandra beschäftigt hatte, wußte ich. Im Sommer 1990 hatte er, am gleichen Ort in München, seinen Vortrag 'Achill. Über den Verlust der Bilder ‘ gehalten. Ein Stück deutscher Prosa, kunst- wie existenzerhellend, das die Gattungskonventionen von Wissenschaft und Kunst weit hinter sich läßt.

Angelika Geyer hat, im gleichen thematischen Zusammenhang, die Spuren des Amazonen-Themas in der antiken Kunst sorgsam aufgesucht. Bernd Leistner, der bisher am weitesten nach vorne gehende Zweifler, damit aber der Kleist am nächsten Kommende unter den Wissenschaftlern der im November 1990 bereits nicht mehr existierenden DDR, kam zu Wort, und Bernhard Böschenstein machte zum Abschluß, auch als Auslöser einer ungemein dichten und kontroversen Diskussion, deutlich, was mit einer gediegenen Epochenüberschau an Einsichten möglich ist.

Die Einladung durch die Bayerische Akademie der Schönen Künste erwies sich als Geschenk unter Freunden; freie Vereinigungen bieten, anders als es der Staat mit seinen Haushalts- und sonstigen Spielregeln vermag, angemessenen Lebensraum. Der Präsident der Akademie, Prof. Dr. h.c. Heinz Friedrich, begrüßte die Teilnehmer: 
Meine sehr verehrten Damen und Herren, als Hausherr der Bayerischen Akademie der Schönen Künste bin ich höflicherweise gebeten worden, Ihnen guten Tag zu sagen. Gern nehme ich die Gelegenheit nicht nur zu dieser Freundlichkeit wahr; ich wünsche Ihnen auch im gleichen Atemzug einen erfolgreichen Tag und einen nicht minder erfolgreichen Abend.

Hausherr: Ich spreche diese Begrüßungsworte weniger als Herr des Hauses, sondern weit mehr als Gastfreund der Kleist-Gesellschaft. Habe ich doch alle oder fast alle Geburtswehen des Kleist-Preises miterlebt, und ich habe auch an allen Schwierigkeiten der ersten Lebensjahre teilgenommen, ja man kann auch sagen: sie miterlitten. Man wußte ja lange nicht, ob dieses Kind lebensfähig sei. Viele Leihmütter reichten ihm in den ersten Lebensjahren die Flasche. Manchmal war ich aufgefordert, diese Flasche und die dazu gehörige Milch nicht nur der frommen Denkungsart, sondern auch der materiellen Substanz zu besorgen, und zwar um Gottes Lohn.

Natürlich fragt man sich, wenn man so etwas tut, warum man es tut und ob Gottes Lohn dafür ausreiche. Nun: Eitelkeit oder Profilneurose bieten hier jedenfalls keinen Ausgleich. Da gibt es lohnendere Objekte als den Kleist-Preis, um sich einer mehr oder minder staunenden Öffentlichkeit zu empfehlen. Man leistet solche Hilfsdienste vielmehr, weil einen die Sache interessiert und fesselt. Man sieht, wie einer - in diesem Falle der Professor Kreutzer - versucht, das Exempel einer Sache zu statuieren, und man fühlt sich herausgefordert, ihm dabei zu helfen, zumal dann, wenn einen, wie gesagt, diese Sache auch noch selbst bewegt, erregt und anregt. Diese Sache heißt Kleist, Erforschung des Phänomens Kleist, aber auch Begegnung mit dem Phänomen Kleist und, was mir besonders wichtig erscheint, Vergegenwärtigung des Phänomens Kleist. Die Kleist-Gesellschaft trägt zu dieser Vergegenwärtigung auf beachtliche Weise bei. Dafür liefert das Symposium heute und morgen schon thematisch einen überzeugenden Beweis. Mythos und Geschichte: ein großes Thema, ein immer wieder bewegendes und nie veraltendes Thema. Auf die Vorträge von heute und morgen und die Aussprachen darüber darf man gespannt sein.

Im Namen der Bayerischen Akademie der Schönen Künste wünsche ich der Tagung der Kleist-Gesellschaft einen produktiven Verlauf und damit viele neue Anregungen. Mit diesem Satz endet die Begrüßungsrolle des Hausherrn. Die Rolle des Freundes und Sympathisanten der Kleist-Gesellschaft jedoch spiele ich gern weiter, solange es diese Gesellschaft und ihren ebenso wagemutigen wie unerbittlichen Präsidenten gibt.

Der Veranstalter selbst hatte dann anschließend ein bißchen näher auf die Konditionen dieser Jahrestagung, die ja ganz neue Elemente enthielt, einzugehen:

Der Name des Dichters Heinrich von Kleist erinnert heute mehr denn je daran, daß die deutsche Dichtung nicht einfach zur geistigen Vorgeschichte der Bundesrepublik rechnet. Zwar, dasjenige, was man in der DDR "Erbeaneignung « nannte, hat man hierzulande ohnehin nicht zu betreiben versucht. Wir haben nun einmal nicht gelernt, die Geschichte unter Besitzkategorien zu betrachten, und das Wort »sich aneignen " trägt überdies unvermeidbar einen dubiosen Doppelsinn. Aber die 
Frage nach der geschichtlichen Heimat dieses Dichters blieb auf besondere Weise offen. Mit einem Hinweis auf die Herkunft Kleists, auf Brandenburg und Pommern, ist noch nicht viel gesagt. Über die deutschen Grenzen von heute müssen wir allemal hinausblicken: ungefähr die Hälfte der bekannteren Werke Kleists stammt in irgendeiner Weise aus Königsberg, und ganz besondere Hoffnung setzte er auf Wien, auch deshalb, weil er sich von seiner 'Hermannsschlacht ' dort auf dem Theater unmittelbare Wirkung versprach. Es sieht wie eine aparte Pointe aus, daß wir des Dichters der >Hermannsschlacht heute in der Residenz eines Rheinbundfürsten gedenken. Aber eben damit hätte er sich am ehesten einverstanden erklären können: als politischer Dichter hatte Kleist die Wirklichkeit des Alten Reichs vor Augen, und wenn er dessen Daseinsrecht verteidigt, mit einer Schroffheit, die uns noch heute erschrecken läßt, dann deshalb, weil er den europäischen Völkerfrieden gestört sah.

Für das, was man später Nation nannte, also für das politische Gemeinwesen, gebrauchte Kleist das schlichte Wort "Gemeinschaft «. Eine solche Gemeinschaft der Deutschen vermochte Kleist sich nur im europäischen Zusammenhang vorzustellen. Einer seiner politischen Essays steht unter der Leitfrage "Was gilt es in diesem Kriege? «, das heißt: 'was ist hier und heute unsere alles entscheidende politische Aufgaber? Die folgenden Sätze, die ich aus diesem Essay zitiere, beschreiben nichts, sie mahnen vielmehr etwas an, wenn Sie so wollen: aus dem Geiste der Utopie. »Eine Gemeinschaft gilt es, deren Wurzeln tausendästig, einer Eiche gleich, in den Boden der Zeit eingreifen [...]. Eine Gemeinschaft, die, unbekannt mit dem Geist der Herrschsucht und der Eroberung, des Daseins und der Duldung so würdig ist, wie irgend eine; die ihren Ruhm nicht einmal denken kann, sie müßte denn den Ruhm zugleich und das Heil aller Übrigen denken, die den Erdkreis bewohnen; deren ausgelassenster und ungeheuerster Gedanke noch, von Dichtern und Weisen, auf Flügeln der Einbildung erschwungen, Unterwerfung unter eine Weltregierung ist, die, in freier Wahl, von der Gesamtheit aller Brüder-Nationen, gesetzt wäre. " Hie und da kommt es noch vor, daß man Kleist einen "preußischen " Dichter nennt. Aber das müßte man schon ganz sorgfältig begründen, zumal vom Kaiserreich bis zum Dritten Reich genug Mißbrauch mit dem Preußen Kleist getrieben worden ist.

Die Mitglieder der Nachkriegs-Kleist-Gesellschaft verteilen sich über die ganze Welt. Das ist völlig anders als bei ihrer 1945 untergegangenen Vorgängerin. Wir sind keine bunderepublikanische Vereinigung, erst recht keine deutsche, und wir werden jetzt nicht etwa zu einer gesamtdeutschen. Japaner, Koreaner, Chinesen, Australier konnten wir immer schon zu unseren Mitgliedern zählen. Jetzt treten Mitbürger aus Sachsen, Pommern und Brandenburg bei. Damit schließt sich eine Lücke, die wir immer schmerzhaft empfunden haben. Vielleicht ist sogar das Wort "Wunde « das bessere, denn es deutet an, daß es Zeit kosten wird, bis sie sich schließt. Formal gesehen wären solche Beitritte immer schon möglich gewesen, aber sie wären nicht gerne gesehen worden. Dergleichen ist nie geradezu verboten worden. Aber ein Sektionsleiter oder dergleichen fand sich immer, der abmahnte. Und was man davon zu halten hatte, das mußte dann ein jeder für sich selbst entscheiden.

Die Kleist-Gesellschaft ist nun einmal bei einem Westberliner Amtsgericht ins 
Vereinsregister eingetragen, und es war der politische Zankapfel Berlin, der die Kleist-Gesellschaft, von der anderen Seite aus gesehen, politisch ungenießbar machte. Wie so vieles war das eine absurd unrealistische Einschätzung der Lage. Am realen Haushalt der Kleist-Gesellschaft ist Berlin mit etwa vier Prozent beteiligt, und die ökonomische Basis ist es, die entscheidet. Erst 1986 konnten sich zum erstenmal auch Autoren aus Weimar und Leipzig im Kleist-Jahrbuch zu Wort melden. Im gleichen Jahr ist freilich der Präsident der Goethe-Gesellschaft, KarlHeinz Hahn - diese Akademie hier hat ihn 1988 mit ihrer Hausenstein-Ehrung ausgezeichnet - noch gemaßregelt worden, weil er, immerhin schon im Rentenalter stehend, sich die Freihheit genommen hatte, im Charlottenburger Schloß bei einer Eröffnungsveranstaltung wie dieser hier einfach zuzuhören. Leicht ist es uns jedenfalls nicht gemacht worden. Aber gerade deshalb haben wir wenig Neigung zu rückwärtsgerichteten Betrachtungen, wir haben jetzt auch keine Zeit dazu. Vor allem aber: unsere Gegenwart sieht, dem Himmel sei Dank, ganz anders aus.

Wir treffen uns bereits zum drittenmal, seitdem sich die politische Landkarte zu verändern begonnen hat, und von $\mathrm{Mal}$ zu Mal hat sich das Klima verbessert. Als Zeitgrenze sehe ich die Tage an, an denen die Menschen auf den Straßen und Plätzen zusammenkamen und dort stehenblieben, obwohl sie Angst hatten. In den letzten Oktobertagen des vergangenen Jahres hatten wir bei einem Kolloquium erstmals Redner aus der DDR unter uns und das, sage und schreibe, in West-Berlin. Und im April dieses Jahres 1990 trafen wir uns unter unwiederholbaren Vorzeichen in der Universität in Budapest. Das Plakat, das die Konstellation für jene Veranstaltung festhält, wird denkwürdig bleiben: Heinrich-von-Kleist-Gesellschaft in Gemeinschaft mit der Eötvös Loránd Universität Budapest, mit Unterstützung des Goethe-Instituts, mit Unterstützung des Kultur- und Informationszentrums der Deutschen Demokratischen Republik und des Stifterverbandes für die Deutsche Wissenschaft. Das werden wir nie wieder erleben, daß uns zwei deutsche Botschafter zu Empfängen einladen. In Budapest ereignete sich der eigentliche Durchbruch. Heute, hier in München, erreichen wir erstmals das Stadium des Selbstverständlichen - im dreißigsten Jahr des Bestehens dieser Gesellschaft.

Wenn uns eine Institution wie die Bayerische Akademie der Schönen Künste gastfreundlich aufnimmt, dann ist das keine beliebige Geschmacksentscheidung, es ist eine echte Symbiose, jedenfalls unter der Bedingung, daß eine literarische Gesellschaft sich auch dem literarischen Leben zuwendet, wie es mit dem KleistPreis geschieht. Frühere Gastgeber waren die Stiftung Preußischer Kulturbesitz, dann das Deutsche Literaturarchiv Marbach, die Freie und Hansestadt Hamburg, damit also ein Bundesland, Frankfurt am Main, also eine Kommune, zweimal der Senat von Berlin. Gäste der Bayerischen Akademie der Schönen Künste sind wir heute zum zweiten Mal: doppelter Dank deshalb, sowohl dem Präsidenten wie auch dem Sekretariat für seine wahrlich große Mühe und Sorgfalt. Es wäre zu hoffen, daß sich auch Kleists Geburtsstadt, Frankfurt an der Oder, in nicht zu ferner Zukunft in die Reihe solcher Gastgeber einfügte. Die nächste Verabredung, für die Vergabe des Kleist-Preises 1991, ist getroffen, mit dem Staatsschauspiel Dresden, also mit der Stadt, in der Kleist seine zweite Geburt, die Geburt des Künstlers, erlebte. 
Ich leite über zum Programm. Die vier Vorträge sollen gemeinschaftlich an Dinge erinnern, die den Dichter Kleist bewegten, als er sich die Konstellationen seiner Werke zurechtlegte. Natürlich hoffen wir, daß es wissenschaftlich gut begründete Vorträge sind, aber es sind nicht die methodischen Kunststücke der Wissenschaft als solcher, die Ihnen vorgeführt werden sollten, dergleichen gehört ins Seminar. Wir wissen im Grunde kaum, wie Kleist über die Kunst dachte. Er äußert sich selten darüber, selten zusammenhängend, und meist auch wieder in der Sprache der Kunst. Aber an einigen Stellen spüren wir: er hat ungeheuer viel gelesen und noch mehr gesehen, er hat ein weites Gedächtnis für die Überlieferung, seine Themen und Gestalten kommen von weither.

Die Veranstaltung hatte eine "Sollbruchstelle", wie der Techniker dergleichen nennt. Das war die öffentliche Verleihung des Kleist-Preises. Vorsichtshalber hatte die Akademie der Künste bei ihrer gleichfalls im Residenzkomplex arbeitenden Schwester, der Bayerischen Akademie der Wissenschaften, deren Sitzungssaal erbeten, mit immerhin bis zu 480 Plätzen gegenüber gut 200 im eigenen Vortragssaal. Aber die Anmeldungen gingen rasch über solche Größenordnungen hinaus. Da war es denn ein besonderer Glücksfall, daß der Herkulessaal der Residenz an diesem Abend nicht vergeben war und daß die Bayerische Schlösserverwaltung rasch und unkonventionell reagierte. Der Akademiepräsident Heinz Friedrich übernahm auch bei dieser Gelegenheit die ihm zukommende Rolle, seine Worte seien deshalb gleichfalls hier festgehalten:

Meine sehr verehrten Damen und Herren, ich habe die Ehre, Sie in meiner Eigenschaft als Präsident der Bayerischen Akademie der Schönen Künste zur Verleihung des Kleist-Preises durch die Kleist-Gesellschaft begrüßen zu dürfen. Daß ich die angebotene Ehre wahrnehme, ist, was den Herkulessaal angeht, nicht mehr ganz protokollgerecht. Ursprünglich sollte die Verleihung des Preises in den Räumen der Bayerischen Akademie der Schönen Künste stattfinden. Inzwischen haben die Säle gewechselt, und sogar zweimal. Herr Professor Kreutzer hat mich gebeten, die Begrüßungsrolle des ursprünglichen Gastgebers auch hier im Herkulessaal zu übernehmen. Die Tagung der KleistGesellschaft 1990 selbst fand und findet - wie vorgesehen - in den Räumen der Bayerischen Akademie der Schönen Künste statt. Die Akademie hat sich gern als Gastgeber angeboten, weil sie sich dieser Gesellschaft eng und freundschaftlich verbunden fühlt. Aber nicht nur dies: Sie unterstützt auch gern die Absichten, die diese Gesellschaft vertritt.

Gesellschaft, Kleist-Gesellschaft wozu? Es gibt inzwischen Gesellschaften dieser Art wie Sand am Meer, von der Mozart-Gesellschaft über die Goethe-Gesellschaft bis hin zur Eichendorff- und anderen Gesellschaften. Oft hat man den Eindruck, als könne ein Autor von Rang, habe er erst das Zeitliche gesegnet, nicht mehr ohne Gesellschaft auskommen, um sich der Nachwelt zu empfehlen. Sogar der geistige Mittelstand etabliert schon Gesellschaften jeglicher Art. Kleist hat sich längst der Nachwelt empfohlen. Braucht er dann überhaupt eine Gesellschaft? Nun: ich 
meine der heutige Abend beweist, daß Kleist eine Gesellschaft braucht, und sei es auch nur, um ein so außerordentliches Ereignis wie die Verleihung des KleistPreises der Öffentlichkeit zu offerieren und damit den Namensgeber des Preises mit einem höchst aktuellen Ausrufezeichen zu versehen.

Gewiß: das Interesse, das Sie heute hier zusammengeführt hat, gilt auch und vor allem dem Preisträger Heiner Müller. Aber spricht das gegen den Kleist-Preis, gegen die Kleist-Gesellschaft? Das Gegenteil ist wohl der Fall. Die Tatsache, daß auf recht außergewöhnlichem Weg der Preisträger für diesen Kleist-Preis ermittelt und ausgezeichnet wird, spricht für den lebendigen Dialog derer, die in dieser Gemeinschaft zusammenkommen und sich vornehmen, zu Ehren eines außergewöhnlichen Dramatikers auch außergewöhnliche Entscheidungen zu treffen.

Der Kleist-Preis steht nicht für literarische Heldenverehrung. Indem die KleistGesellschaft den Kleist-Preis verleiht, gibt sie zu erkennen, daß für sie Kleist mehr ist als nur ein Forschungs- und Verehrungs-Objekt. An Kleists Genie erprobt und überprüft sie aktiv die Dramatik der Gegenwart. Wer sich dem Anspruch dieses Preises stellt und ihm standhält, den trifft ein Ritterschlag besonderer Art. Die Bayerische Akademie der Schönen Künste rechnet es sich als große Ehre an, daß sie hier in München quasi die Schirmherrschaft über die Herbstveranstaltung der Kleist-Gesellschaft und damit auch für den heutigen Abend übernehmen darf.

Im Rückblick markiert die Münchner Tagung 1990 einen geschichtlichen Einschnitt. Die Mitglieder gedachten zweier Verstorbener, der letzten, die noch aus der geistigen Tradition Berlins kamen, aus der die Kleist-Gesellschaft einmal entstanden ist: der Verlegerin Dr. Ellinor Kahleyss und des langjährigen Präsidenten der KleistGesellschaft, ihres späteren Ehrenpräsidenten, Prof. Dr. Wieland Schmidt.

Hans Joachim Kreutzer 